\title{
DIE GESELLSCHAFTLICHE EINBETTUNG HISTORISCHER GÄRTEN UND IHRE KLIMAWANDEL-RESILIENZ ${ }^{1}$
}

\begin{abstract}
Der Beitrag fokussiert aus einer soziologischen Perspektive auf die vielfältige Einbettung der die historischen Gärten verwaltenden Organisationen und unterscheidet zwischen kognitiver, kultureller, struktureller und politischer Embeddedness. Mit Methoden der qualitativen Sozialforschung und auf Basis eines explorativ erhobenen Interviewmaterials zeigen wir, dass die Resilienzstrategien der Gärten im Umgang mit (Klima-)Risiken auch von ihrer jeweiligen Einbettung abhängen.
\end{abstract}

This article focuses from the sociological angle on the diverse embeddedness of the organisations that manage historic gardens and distinguishes between cognitive, cultural, structural and political embeddedness. Using social research methods and explorative interview material we demonstrate that the resilience strategies of gardens when dealing with the (climate) risk likewise depend on their respective embeddedness.

\section{Einleitung}

Das für die interdisziplinäre Arbeitsgruppe leitende Erkenntnisinteresse zielt auf die Herausforderungen des Klimawandels für die historischen Gärten und ihren Umgang damit. Die im vorliegenden Beitrag angestrebte soziologische Auseinandersetzung mit der Thematik muss sich im ersten Zugriff die Frage gefallen lassen, welche Relevanz eine sozialwissenschaftliche Betrachtung bei einem primär als ökologische Herausforderung gerahmten Phänomen wie dem Klimawandel erlangen kann.

Im Text werden Maskulinum und Femininum verwendet, wenn es um Personen geht. Gemeint sind grundsätzlich alle Menschen, gleich welcher Geschlechtsidentität sie sich zugehörig fühlen.

1 Wir danken der interdisziplinären Arbeitsgruppe »Historische Gärten im Klimawandel « und Richard Münch für die konstruktiven Hinweise, der Berlin-Brandenburgischen Akademie der Wissenschaften für die finanzielle Unterstützung des Vorhabens und allen InterviewpartnerInnen. 
Zur Relevanz soziologischer Erkenntnisse. Der Klimawandel ruft zunächst zu einer naturwissenschaftlichen Bearbeitung auf. Die ist jedoch unseres Erachtens nicht ausreichend, um zu einem umfassenden und nachhaltigen Maßnahmenkatalog zu gelangen. Die aus naturwissenschaftlicher Sicht identifizierten Aktivitätserfordernisse für einen adäquaten Umgang mit den anstehenden Herausforderungen müssen auch umgesetzt werden können. Wir sehen am Beispiel des Großen Tiergartens in Berlin, dass das eloquenteste Parkpflegewerk keine Wirkung entfalten kann, wenn Politik, Verwaltung und Gesellschaft nicht auch auf seine Umsetzung drängen. Damit die Organisation und das Management historischer Gärten angemessen auf den Klimawandel reagieren können, benötigen sie vielfältige Unterstützung sowohl durch ihre externen Stakeholder (etwa AnwohnerInnen, PolitikerInnen) als auch durch ihre internen Anspruchsgruppen (zuvorderst ihrer MitarbeiterInnen). Über ein Stakeholder-Management hinausgehend gehört zur Analyse der organisationalen Umwelt historischer Gärten aber auch eine gewisse Erkenntnisoffenheit dahingehend, implizite, unhinterfragte Narrative, Wertungen oder Normen aufzudecken und durch ihre Infragestellung im besten Fall zu erfolgversprechenderen und kreativeren Lösungen zu gelangen und dafür Unterstützung zu mobilisieren. Sich der Begrenztheit der eigenen Wahrnehmung bewusst zu werden, ist ein durchaus lohnenswertes, aber mühsames Unterfangen, nicht nur für Individuen, sondern auch für Organisationen.

Leitende Fragestellung des Beitrags. Vor diesem Hintergrund modifizieren wir im Sinne einer Komplementierung naturwissenschaftlicher Forschungsansätze das übergreifende Erkenntnisinteresse der interdisziplinären Arbeitsgruppe wie folgt und fragen: Wie können historische Gärten mit Hilfe des soziologischen Konzepts der Embeddedness resilienter gegenüber den Herausforderungen des Klimawandels werden?

Resilienz historischer Gärten. Der mittlerweile von verschiedenen Disziplinen okkupierte Begriff der Resilienz hat seinen Ursprung in der Forschung zum Umgang mit ökologischen Krisen und wird seither in den Disziplinen weiterentwickelt. Wir nutzen als Arbeitsdefinition einen Diskussionskontext, in dem Resilienz verstanden wird »als die Fähigkeit von sozial-ökologischen Systemen, kontinuierlich fortzubestehen und sich gegebenenfalls wieder neu zu organisieren « (Deppisch 2016, 201). Übertragen auf die Geschicke der Gärten geht es um die Etablierung einer Strategie, die mit den Herausforderungen des Klimawandels in einer Weise umzugehen vermag, die letztlich die historischen Gärten als Erbe auch für die nächsten Generationen in seiner ursprünglichen Form erhalten kann. Die Entwicklung einer Resilienzstrategie und der dorthin führende Prozess einer Transformation stellen sich dabei für jeden Garten unterschiedlich dar.

Keine Resilienzstrategie, egal für welches Problem, kommt umhin, sich in produktiver Weise mit den gegebenen organisationalen Kontextbedingungen auseinanderzusetzen. Organisationen, auch die Organisationsformen historischer Gärten, sind eingebettet in vielfältige Bezüge zu ihrer organisationalen Umwelt. Die Möglichkeitsräume für einen transformativen Wandel hin zu mehr Resilienz werden im Lichte unterschiedlicher Einbettungen unterschiedlich gedacht und bewertet. Beispielsweise empfinden manche Gärten die Einbindung und Betreuung von Ehrenamtlichen als zusätzliche Belastung für das ohnehin 
überlastete Bestandspersonal, etwa weil das Einweisen der freiwilligen Kräfte wertvolle Zeit beansprucht, andere wiederum sehen sie gerade bei alläglichen Abläufen als hochwillkommene Unterstützung des überlasteten Bestandspersonals. Die einen verzichten dementsprechend auf das Akquirieren von Ehrenamtlichen, die anderen fördern und feiern sie (A18, B7, D16, 24, F21, 22).

Wir wollen im Folgenden die Einbettung historischer Gärten genauer beleuchten und dafür das soziologische Konzept der Embeddedness fruchtbar machen. Wir erhoffen uns davon sowohl Anregungen für einen neuen Blick auf eine Ist-Analyse bereits vorhandener Klimawandel-Strategien und die etwaige Aufdeckung von Verbesserungspotentialen als auch - abstrahierend gedacht - eine allgemeine Sensibilisierung für mögliche Ursachen bisheriger Konflikte und Verhärtungen, die sich vielleicht mit der soziologischen Brille zu unterschiedlichen Einbettungen der Akteure besser verstehen und leichter einordnen lassen.

\section{Theoretischer Rahmen}

Embeddedness. Organisationen werden in der modernen Organisationssoziologie als per se offene und in vielfältigen Wechselbeziehungen mit ihrer gesellschaftlichen Umwelt stehende Einheiten konzipiert (Scott/Davis 2007). Mit dem auf diesem Grundverständnis aufbauenden Konzept der Embeddedness gelingt es, die Qualität und Wirkung einer solchen Verankerung von Individuen oder Organisationen in ihrer jeweiligen sozialen Umwelt in den Blick zu nehmen. Das können die losen Kontakte mit dem dennoch entscheidenden Hinweis etwa für die Pflanzenpflege sein, die kulturell geprägten Wertvorstellungen von idealen Karriereverläufen, die durch die Professionstätigkeit eingeübten Lösungsschablonen für den Umgang etwa mit Schädlingen oder die Akzeptanz von formalen und informalen Forderungen nach Natur- oder Denkmalschutz ${ }^{2}$, die in ihrer Vielfalt auf das eigene Entscheidungsverhalten einwirken. Einbettung ermöglicht in positiver Lesart den Aufbau von Vertrauen, von passgenauem Informationstransfer oder gemeinsamer Problemlösung (Uzzi 1996). Sie kann aber auch limitierend für die Zielerreichung sein, oder auch die Zielsetzung verändern, wenn etwa der nach der Wende herrschende Diskurs einen sehr schnellen Rückbau der Mauer im Park Babelsberg forderte, wohingegen spätere Herangehensweisen den Aspekt der Erinnerungskultur bevorzugt und einige Mauerreste als Mahnmal erhalten hätten.

Der Ansatz von Zukin und DiMaggio. Wir bauen in unserem Beitrag auf das vielbeachtete Konzept von Zukin und DiMaggio (1990) auf, die im Sinne einer Synthese bereits vorhandener Ansätze (allen voran Granovetter 1985) eine Weiterentwicklung anbieten. Während sie ihr Konzept ursprünglich für ein besseres wirtschaftssoziologisches Verständnis ökonomischer Entscheidungsfindung entwickelt haben, lässt es sich auch für Entscheidungsverhalten in nicht-wirtschaftlichen Kontexten fruchtbar machen.

2 Siehe den Beitrag von Tobias Plieninger in diesem Band. 
Die vier Dimensionen der Embeddedness nach Zukin und DiMaggio. Zukin und DiMaggio (1990) etablieren eine analytische Trennung der Embeddedness in vier Dimensionen: kognitiv, kulturell, strukturell und politisch. Die kognitive Embeddedness geht davon aus, dass die Entscheidungsfindung von Individuen und Organisationen nicht vollständig rational erfolgt, sondern limitiert und beeinflusst ist, etwa durch begrenzte Wahrnehmungskapazitäten oder dominierende Interpretationsmuster. Akteure haben entweder aufgrund der Überkomplexität der Realität nicht alle Informationen zur Verfügung oder blenden im Sinne einer bounded rationality bewusst oder unbewusst Informationen aus, um zu einer Entscheidung zu gelangen. Die kulturelle Einbettung bezieht die Wirkmächtigkeit von geteiltem gemeinsamem Verständnis mit ein. Die in der jeweiligen (Sub-)Kultur vorherrschenden Sinnzuschreibungen, Werte und Normen, Ideologien, unhinterfragten Annahmen oder formalen Regelsysteme prägen Zielvorstellungen und Handlungen. Die strukturelle (oder auch relationale) Embeddedness richtet ihr Augenmerk auf die wiederkehrenden und anhaltenden zwischenmenschlichen Beziehungen und inter-organisationalen Kontakte. Die Muster der vorzufindenden Beziehungsgeflechte und ihrer jeweiligen Ausgestaltungen (z.B. kooperativ versus konkurrenzorientiert) werden als Nährboden für Vertrauen, Loyalität, Solidarität oder geteilte Identitäten gesehen, die etwa Professionsgruppen verbinden oder Friktionen heraufbeschwören können. Die politische Einbettung berücksichtigt die institutionellen Kontexte, in denen Entscheidungen gefällt werden, etwa von Gesetz, Rechtsprechung und Verwaltungsrichtlinien, aber auch die zuweilen vagen Leitlinien einer politischen Agenda. Sie berücksichtigt zudem alle Ausprägungen von Machtasymmetrien und -dynamiken.

Die Aufteilung der Embeddedness in die vier Dimensionen ist lediglich als analytische Trennung zu verstehen, für die in der Realität in der Regel keine eineindeutige Zuordnung möglich ist. Auch die gegenseitige Beeinflussung und die vielfältigen Wechselwirkungen zwischen den Dimensionen sind durch diese analytische Differenzierung nur ansatzweise $\mathrm{zu}$ erfassen.

In den nächsten Schritten werden wir diese vier Dimensionen ausführlicher vorstellen und auf ihre Relevanz im Hinblick auf historische Gärten prüfen. Daran anschließend erfolgt eine explorative Illustration der jeweiligen Dimensionen durch Auszüge aus den geführten Interviews oder ihre Paraphrasierungen und das weitere empirische Material. Dazu stellen wir zunächst unser methodisches Vorgehen zur Erhebung vor.

Methodisches Vorgehen. Die vorliegende explorative Studie versteht sich als Auftakt für weiterführende Forschung. Im Jahr 2017 wurden acht leitfadengestützte Interviews mit elf Führungskräften (w/m) oder ExpertInnen in einflussreicher Position in ostdeutschen historischen Gärten geführt, deren Länge zwischen etwa einer und drei Stunden variierte (insgesamt 15 Stunden und 10 Minuten). Wir haben allen Interviewten vollständige Anonymität zugesichert, die wir nur mit explizitem Einverständnis selektiv aufheben. ${ }^{3}$ Die 
Interviews waren konzeptionell methodisch zweigeteilt. Zum einen wurde ein klassisches Leitfadeninterview geführt, zum anderen wurden die InterviewpartnerInnen gebeten, eine von uns vorbereitete fallspezifisch abgestimmte Auswahl an internen und externen Anspruchsgruppen zueinander in Beziehung zu setzen. Dies erfolgte über das Auslegen von Stakeholder-Kärtchen auf dem Tisch. Diese Aufgabenstellung provozierte den für uns relevanten Erzählanreiz, der wertende und beschreibende Elemente hervorbrachte. Die Erläuterungen wurden häufig mit Anekdoten verbunden, die die Ereignisse illustrierten. Nicht alle InterviewpartnerInnen waren gleichermaßen bereit, hatten die zeitlichen Ressourcen oder räumliche Ausstattung, beide Teile des Interviews durchzuführen. Ein/e InterviewpartnerIn bevorzugte einen gänzlich frei-assoziativen Erzählfluss. Bis auf ein Interview durften alle aufgezeichnet und transkribiert werden.

Diese Darstellungen des Beziehungsgeflechts und der Rahmenbedingungen ermöglichen uns Rückschlüsse auf die Einbettung der Organisationseinheit »historischer Garten« und die variantenreichen Darstellungen der jeweiligen Gestaltungsräume. Unsere Methode zielt primär auf die Wahrnehmungen, Deutungen und Erklärungsangebote der InterviewpartnerInnen. Diesem Vorgehen liegt die Annahme zu Grunde, dass die interviewten Führungskräfte und einflusshabenden ExpertInnen die Herausforderungen des Klimawandels aus ihrer Deutung der organisationalen Einbettung heraus angehen werden. Die Auswertung der Interviews erfolgte unter Berücksichtigung der Intercoder-Reliablilität durch die Autorin und den Autor in Anlehnung an die Inhaltsanalyse nach Mayring (2015).

Ergänzend haben wir durch die Analyse von Mediendebatten, die Teilnahme an öffentlich zugänglichen Fachtagungen, an geführten Exkursionen im Rahmen der interdisziplinären Arbeitsgruppe und das freie Besuchen im Touristenmodus sowie durch teilnehmende Beobachtung und informelle Gespräche mit Parkführungspersonal, AnwohnerInnen, Mitgliedern in Freundeskreisen oder ehrenamtlich Engagierten weiteres Hintergrundmaterial gesammelt. Das trotz des explorativen Charakters vergleichsweise gehaltvolle und umfangreiche Material kann in diesem kurzen Beitrag leider nur in Ausschnitten präsentiert werden.

Der Embeddedness-Ansatz und seine Anwendung auf die Situation historischer Gärten in Zeiten des Klimawandels. Kein historischer Garten ist wie der andere, die Varianz allein zwischen den historischen Gärten in Ostdeutschland ist immens. Für eine Annäherung an unseren Untersuchungsgegenstand ist es instruktiv, ein paar schlaglichtartige Unterscheidungskriterien aufzuzeigen, wohlwissend, hier keine abschließende Systematisierung anbieten zu können. Einige Gärten sind vorwiegend Besuchsgärten, für die auch lange Anfahrtswege in Kauf genommen werden, einige werden vornehmlich von AnwohnerInnen frequentiert. Dies resultiert zum Teil auch aus der Lage in bzw. an einer Stadt oder in einer ländlichen Gegend mit je unterschiedlicher Sozialstruktur der AnwohnerInnen. Das

spezifischen Bezeichnungen (etwa von Gremien), sondern beschreiben sie mit Allgemeinbegriffen. Die Legenden A, B, C sind nicht durchgängig einer/einem Interviewten zugeordnet. Zum Teil erhalten Interviewte verschiedene Kennzeichnungen, damit sich nicht über die Zusammenführung aller Aussagen ein dann doch zuordenbares Gesamtbild ergeben kann. In diesem Sinne wurde auch Dialektales ins Hochdeutsche übertragen oder die Nutzung der Begriffe »Park« und »Garten« bewusst unsystematisch verwendet. 
freiwillige Engagement für die Gärten im Ehrenamt, in Freundeskreisen, durch ErbenspenderInnen usw. variiert von fast nicht existent bis sehr groß. In ihrer institutionellen Verfasstheit reicht die Varianz in Ostdeutschland vom Modell öffentlich-rechtlicher (Landes-) Stiftungen über nichtselbständige kommunale Stiftungen bis hin zu einer Integration in die Verwaltung der sonstigen Grünflächen der Stadt, zur Zusammenführung der zuvor aufgeteilten Verantwortlichkeiten in einer landeseigenen Behörde, zu gemeinnützigen GmbHs mit je unterschiedlichen Finanzierungsmodellen von Kommune, Stadt, Land und Bund, und ergänzt durch Fördermittel und private Spenden. Anders als einige historische Gärten in Westdeutschland sind die Gärten in Ostdeutschland zumeist nicht in Privatbesitz, aber durch die Restitution von nach 1945 enteigneten mobilen Kunst- und Kulturgütern im Zuge der deutschen Einheit zuweilen noch mit ihren ehemaligen EigentümerInnen verbunden. Einige wenige Gärten sind weltweit bekannt, andere werden im Verbund mit anderen Sehenswürdigkeiten national oder sogar international wahrgenommen; die meisten genießen vor allem lokal oder regional Aufmerksamkeit. Der Status als UNESCO-Weltkulturerbe sichert manchen Parks einen festen Platz auf der Reiseroute vielfach auch internationaler KulturtouristInnen. Einige Gärten sind als geographischer Solitär entstanden, andere sind Teil einer größer angelegten Gartenlandschaft. Die Parks konkurrieren um regionale Aufmerksamkeit, auch mit anderen Sehenswürdigkeiten, oder gehen kooperative Beziehung miteinander ein.

Nach dieser Andeutung zu den vielfältigen Verfasstheiten von Gärten werden wir im nächsten Schritt die vier Dimensionen der Embeddedness mit den Erkenntnissen aus den Interviews in Beziehung setzen.

Die kognitive Embeddedness. Diese Dimension der Einbettung thematisiert die Limitierungen, denen EntscheiderInnen regelmäßig unterliegen. Ihre Entscheidungen basieren oftmals auf begrenzten und unvollständigen Informationen, werden in Situationen hoher Komplexität und unter Zuhilfenahme von Vorannahmen, Stereotypen, Heuristiken oder unhinterfragten Werten gefällt. Der Aspekt der kognitiven Embeddedness lenkt die Aufmerksamkeit auf die begrenzten Fähigkeiten von Individuen oder Organisationen, rationale Entscheidungen zu treffen (Zukin/DiMaggio 1990, 15ff). Um entscheidungsfähig zu bleiben, ignorieren EntscheiderInnen oftmals bewusst oder unbewusst Informationen. Führungskräfte streben im organisationalen Entscheidungsprozess in solchen Situationen gerne eine befriedigende, aber nicht unbedingt optimale Entscheidung an (March/Simon 1958). Dem wollen wir den Einfluss von Idiosynkrasien und des Selbstverständnisses von Führungskräften hinsichtlich der letztlich gefällten Entscheidungen hinzufügen. In der Summe kann es schwerwiegende Konsequenzen haben, wenn eine Vielzahl an Akteuren im Feld ihren je unterschiedlichen Begrenzungen in der Entscheidungsfindung unterliegt und letztlich Entscheidungen großer Tragweite gefällt werden, die nicht situationsangemessen oder nachhaltig im Umgang mit den Herausforderungen für die Gärten sind.

Die Implikationen kognitiver Embeddedness für Führungskräfte historischer Gärten in Zeiten des Klimawandels sind offensichtlich. Prognosen sind geradezu idealtypisch durch einen hohen Grad an Unsicherheit und Komplexität charakterisiert. Der Klimawandel kann 
sich als plötzliche, kaum vorhersagbare Krisensituation in Form eines heftigen Sturms oder eines Hochwassers zeigen. Noch herausfordernder für EntscheiderInnen sind die Auswirkungen der schleichenden Prozesse, die nicht immer eindeutig dem Klimawandel zugeschrieben werden können. Die trockenen Sommer, der Hitzestress, die zu warmen Winter oder die verschobenen Jahreszeiten sind einige der thematisierten Umweltveränderungen, die sich negativ auf historische Gärten auswirken.

In den Gesprächen wurde deutlich, dass alle EntscheiderInnen gut etablierte Verfahren entwickelt haben, um etwa nach einem Sturm aufzuräumen und die Verkehrssicherung ${ }^{4}$ für das Publikum zügig wiederherzustellen. Weniger eindeutig zeichnete sich in den Gesprächen das Bild, wenn die Sprache auf die langfristigen, schleichenden Prozesse des Klimawandels kam. Die Bandbreite könnte hier kaum größer sein. Die Stiftung Preußische Schlösser und Gärten Berlin-Brandenburg und maßgeblich ihr ehemaliger Generaldirektor Hartmut Dorgerloh hat in Kooperation mit dem Gartendirektor Michael Rohde als Vorreiter das Thema Klimawandel im organisationalen Feld der historischen Gärten mittels Konferenzen, Publikationen und Forschungsprojekten überregional sichtbar auf die Agenda gesetzt ${ }^{5}$. Wir interpretieren dies als eine Reaktion auf die im Kontext kognitiver Embeddedness wahrgenommene Unsicherheit und Komplexität, die die Gestaltungsspielräume auslotet und die identifizierten Limitierungen durch die Hinzuziehung von weiteren wissenschaftlichen und praktischen ExpertInnen proaktiv angeht und gesellschaftspolitisch relevante Themen bearbeitet. Wir greifen aus unseren Ergebnissen eine Positionierung heraus, die der zuvor beschriebenen fast diametral entgegensteht und damit die Spannbreite im Feld veranschaulicht. Auf die Frage nach der Relevanz des Themas Klimawandel für die Gärten, wurde erläutert: »... bei manchem bewährt es sich schlicht und einfach auch, abzuwarten, was passiert, und erst, wenn es sich wirklich verfestigt, dann verändernd einzugreifen ... aber ich würde nie vorausschauend ... dem Klimawandel vorauseilen, eher begleiten ... und manchmal hinterherlaufen ...«(G26). Die kognitive Embeddedness, so unsere Interpretation, führt bei dieser Herangehensweise gerade nicht zu einer proaktiven Bearbeitung, sondern zu einer Bestätigung, dass in Zeiten hoher Unsicherheit und Komplexität eine gewisse Schicksalsergebenheit angemessen sei.

Auch die Überwindung der mit dem Klimawandel einhergehenden Unsicherheiten durch die engere Einbindung der Wissenschaft wird von einigen Akteuren im Feld skeptisch beurteilt. »Auch ihr Projekt ist ja ganz stark fixiert oder orientiert darauf, was müssen jetzt die Gärten machen, um klimafit zu werden ... und ich denke aber, das ist wieder so eine Richtung, ... die so Schnellschuss-to-do-Listen entwickelt, die wir [PraktikerInnen] dann ganz schnell wieder beiseitelegen [und] sagen: >Na ja, jetzt haben wir das Projekt erledigt«« (F28). Dem wird das bereits als Ressource vorhandene Erfahrungswissen der GärtnerInnen gegenübergestellt. »Ich denke, dass [der Klimawandel] manchmal für Gärtner

4 https://naturschutz-und-denkmalpflege.projekte.tu-berlin.de/media/pdf/Hilsberg_Rechtsgutachten_ Endv_Nov2011.pdf (18.07.2018).

5 Vgl. die Einleitung zu diesem Band. 
nicht so überraschend kommt, weil Gärtner über Generationen und Jahrzehnte und Jahrhunderte ja gewöhnt sind an Veränderungen« (G30). »Wir können ja eigentlich auf alles reagieren, und wir können mit jeder Herausforderung auch früher oder später umgehen. Und wir können auch die Mittel dafür früher oder später rekrutieren. Aber es muss eben kompetentes Personal dabei sein« (F28).

Wir haben auch mehrfach Positionen angetroffen, in denen die Existenz des Klimawandels in Frage gestellt wurde, wie etwa im folgenden Zitat: »Ich bin mir auch nicht sicher, ob das, was wir jetzt als Auswüchse des Klimawandels bezeichnen, wirklich damit zu tun hat ... also, das gab es immer « (A27, auch D26, G26). Verwiesen wird auf die zeitgenössischen Beschreibungen von Extremwettereignissen schon zu Zeiten der Parkerschaffer.

Die kulturelle Embeddedness. Diese Dimension der Einbettung bezieht sich auf die in einer (Sub-)Kultur geteilten kollektiven Deutungen, wie Glauben, Normen, Ideologien, Annahmen oder Regelsysteme, die die Wahl der Strategie oder die Definition eines Ziels meist unhinterfragt und unbemerkt beeinflussen (Zukin/DiMaggio 1990, 17). Kultur limitiert wirtschaftliches Handeln, wenn bestimmte Güter, etwa Organe, aus moralischen Gründen als nicht handelbar angesehen werden. Im Umkehrschluss liefert Kultur aber auch diejenigen Normen, Werte und Ideologien, die Handeln als legitim definieren und etwa die Schutzwürdigkeit historischer Gärten als Erbe der Menschheit rahmen. Die Interpretation dessen, was als integres Handeln angesehen wird, kann auch dann Verhalten steuern, wenn Betrug oder abweichendes Verhalten mit geringer Sanktionsgefahr möglich wäre. Die doch nicht genutzte Fahrradwegabkürzung über die Wiese ist ein Hinweis auf den Respekt vor in dem Moment nicht sanktionierten Parkordnungen. »Hier gibt es noch so ein Bürgertum, was die Werte auch noch achtet. Also, wir haben auch mit Vandalismus wenig zu tun« (A23).

Kulturelle Embeddedness prägt natürlich auch den Umgang mit dem Thema Klimawandel. Besonders eindrücklich ist uns dies in den vielfältigen Gesprächen in der Lausitz begegnet. »In Berlin wird gerade gestritten über die Klimaschutzziele der nächsten Legislatur, und davon wird die Lausitz in jedem Fall erheblich betroffen sein, weil, die hängen hier immer noch voll an der Kohle ..., die hoffen alle, es geht noch viele Jahrzehnte weiter « (H9). Und weiter heißt es: »Hier ... spielt Klimawandel überhaupt keine Rolle. Weil über Umweltthemen redet man hier nicht gerne. Das hat mit der Braunkohle zu tun« (H27). »Sie werden hier keine Lobby finden, die sich dafür engagiert.« (H28) »... ich merke jetzt bei mir selber, ich habe gerade die Presse gestern gemacht zu den [aktuellen Sturm-]Schäden: Klimawandel kam bei meiner Erklärung nicht vor« (H28). Andererseits wird positiv hervorgehoben, dass das Braunkohleunternehmen LEAG »halt wichtig für die Lausitz [ist], weil die eben auch Sportvereine, kulturelles Brauchtum, ... überall sind die drin mit ihren Förderungen« (H22). Wir haben im Zuge unserer Forschung auch Gespräche mit sehr engagierten und gutverdienenden LEAG-MitarbeiterInnen geführt, die sich mit Herzblut für den Förderverein des Gartens engagieren. Die Unterstützung für den Garten in der Region ist da, aber sie kann offensichtlich durch die kulturelle Einbettung nicht über das Signalwort Klimawandel mobilisiert werden. 
Die strukturelle Embeddedness. Diese eng an den ursprünglichen Ansatz von Granovetter (1985) anschließende Vorstellung von Einbettung betrachtet die Struktur und Qualität sozialer Beziehungen in den verschiedenen Varianten netzwerkartiger Konfigurationen. Die sich entwickelnden Muster anhaltender sozialer Interaktion lassen sich genauso auf der Mikroebene der Individuen als Beziehungsgeflechte analysieren wie auf der Mesoebene zwischen Organisationen oder zwischen Organisationen und Individuen. Die relevanten Beziehungsgeflechte sind sowohl im Formalen als auch im Informalen zu finden (Zukin/ DiMaggio 1990, 18ff). Die Qualität der Beziehung kann sehr unterschiedlich ausgeprägt sein, sie kann etwa kooperativ oder wettbewerblich, eng oder lose oder von unterschiedlicher Emotionalität geprägt sein (Granovetter 1985; Bandelj 2009).

Unsere Frage nach dem Beziehungsgeflecht und den relevanten Akteuren hat uns nicht nur deutlich mehr Varianz aufgezeigt, sondern auch Hinweise auf die teils sehr unterschiedliche Denkweise gegeben. Zwei InterviewpartnerInnen hatten sofort ein inneres Bild ihrer Stakeholder vor Augen und versuchten, dies bestmöglich mittels der vorgelegten Stakeholder-Kärtchen zu visualisieren. Die meisten waren zunächst von der Fülle der Stakeholder ihres Gartens überrascht und haben dann mit uns im Gespräch und Zug um Zug ein geordnetes Beziehungsgeflecht entwickelt, sind meist aber nicht auf eine sie wirklich überzeugende oder gar vollständige Darstellung gekommen. Auch diejenigen, die ihr Akteursfeld ohne diese visuelle Unterstützung beschrieben haben, waren unterschiedlich systematischanalysierend. Wir konnten eine - gewiss lose - Korrelation feststellen, dass die Fähigkeit zu einer systematischen Reproduzierbarkeit des Stakeholder-Tableaus mit dem Gedanken an die Notwendigkeit einer vollständigen Beziehungspflege aller Stakeholder einherging (wer sich ständig mit allen beschäftigt, kennt sie auch). In entgegengesetzten Fällen konnten wir beobachten, dass ein Ad-hoc-Strukturieren tendenziell auch mit der Überzeugung einherging, dass die Konzentration auf (teilweise sehr) wenige, wichtige Stakeholder zielführend sei, nicht aber die Beziehungspflege in der ganzen Breite. Wir wagen die These, dass die Parks umso resilienter mit den Herausforderungen des Klimawandels umgehen können, je systematischer die Verantwortlichen ihre Anspruchsgruppen wahrnehmen und auf sie zugehen.

Auch wir waren von der Vielfalt der Anspruchsgruppen je Garten und der Unterschiedlichkeit zwischen den Gärten beeindruckt, die ohne Weiteres mit der von mittleren oder größeren Unternehmen vergleichbar ist. Die Herausforderungen des Perspektivwechsels zwischen den Stakeholdern wurde von einer/einem GesprächspartnerIn besonders pointiert formuliert als »wir haben lauter missionarische Überzeugungstäter, die sehr sektoral denken ... so argumentieren in Extremen ... also, dieses Hineindenkenkönnen in eine andere Perspektive ist ganz schwierig « (E17).

Für die Beschreibung der Qualität der Beziehungen greifen wir aus unseren Ergebnissen den Aspekt der Emotionalität mit seiner engen Verbindung zu den Schwierigkeiten des Perspektivwechsels heraus, der uns jeweils recht ausführlich erläutert wurde und für gelingende und scheiternde Kooperationen teils maßgeblich verantwortlich gemacht wird. »Da habe ich auch gedacht, das geht viel schneller, habe aber den menschlichen Faktor wirklich 
unterschätzt« (A9). Die Gärten werden zwar als positiv erlebter, sogar als identitätsstiftender Ort für die Region beschrieben: »[Der Garten] ist für die Leute was, da geht man auch am Wochenende hin, oder wenn die Familie eine Feier hat, ... das wird wertgeschätzt, ja. ... Einschulungsfeier, alles so was« (A9, 25f, auch B12). Zugleich wird den AnwohnerInnen aber auch »ein Wahnsinnsanspruchsdenken « attestiert: »Mein Wille zählt. Ich bin der Bürger. Ich will den Park nutzen« (A24, auch B9, E17). Daher erweise sich Kooperation auch als schwierig. Der Versuch der Vermittlung einer gartendenkmalpflegerischen Perspektive etwa über Podiumsdiskussionen wird ernüchtert beschrieben als Auftritt mit »Kampfansage« der Parknutzer mit Forderungen nach Grillen, Fahrradfahren usw. (B9). Daneben beschreiben einige den hinderlichen Neid auf die Gärten und ihre öffentliche Förderung, wenn ihnen etwa regelmäßig entgegnet würde: »Denen geht es viel zu gut ... Bei euch werden sogar die Gartenzäune vergoldet « (A23, 29, entsprechend B22). »Die sind so satt, die können ja kaum noch laufen, sie müssen sich gar nicht mehr richtig anstrengen « (E17). Aber auch die überregionale Kooperation gestaltet sich unterschiedlich schwierig und ist zum Teil durch regionale Antipathien geprägt, etwa wenn durch die gute Entwicklung in einem Bundesland oder einer anderen Stiftung nach der Wende »so eine gewisse Arroganz« an den Tag gelegt werden würde und als »Hoho! Wir! ... und da waren die nicht sehr kooperativ« charakterisiert wird (A10). Die Liste kann über alle Interviews hinweg beliebig erweitert werden, Stadt gegen Land, AnwohnerInnen versus TouristInnen, AnwohnerInnen gegen Stiftung usw. (E17).

Wir wollen mit diesen Befunden nicht behaupten, die emotionale Aufladung von Interessengegensätzen in den Beziehungsgeflechten von Gärten sei stärker als in anderen Bereichen unserer Gesellschaft - sie rangiert vermutlich in einem ähnlichen Bereich. Wir haben es aber hier mit dem Thema Klimawandel mit einem hochkomplexen Problem zu tun, dem über eine gelingende Kooperation zwischen allen Akteuren begegnet werden kann. Die Erkenntnis, dass mehr Vermittlungsangebote ein wichtiger Schritt sind, ist uns vielfältig begegnet, aber auch der Hinweis, dass »die Kraft« fehlte, etwa auf Grund der schlechten Personalsituation (B10). Vielleicht verfügen die Gärten dennoch dafür über besondere Potentiale im Vergleich zu anderen Feldern. Sie berühren Menschen in besonderer Weise und können vielleicht darüber vergleichsweise leichter Brücken zwischen gegensätzlichen Interessen bauen und UnterstützerInnennetzwerke mobilisieren: »Dieser Park öffnet die Herzen. Das ist so. Das ist ein klarer Befund. Und insofern glaube ich, dass das anhält« (A31). "Was motiviert die [Ehrenamtlichen]? Wirklich die Liebe zum Park irgendwie« (D16).

Die politische Embeddedness. Zukin und DiMaggio (1990, 20ff) operieren mit einem erweiterten Politikbegriff und thematisieren das weite Feld der Machtkämpfe zwischen Akteuren. Sie wollen mit der politischen Embeddedness all diejenigen Beeinflussungen von Entscheidungshandeln einfangen, die auf ungleiche Machtverteilung zurückgeführt werden können.

Wir wenden uns hierfür dem Beispiel des Großen Tiergartens in Berlin zu. Seine Verfasstheit verdeutlicht die dramatischen Auswirkungen der »Zersplitterung der Zuständigkeiten« (C28) als Ergebnis von ungelösten Machtkonflikten und mehrfachen Verantwort- 
lichkeiten (J7) ohne klare Governance-Struktur. Obgleich der Große Tiergarten durch das Straßen- und Grünflächenamt des Stadtbezirks Mitte verwaltet wird, mischen andere Stellen etwa bei der Durchführung von Events mit; es gibt die Zuständigkeit des Ordnungsamts und Interessenkonflikte zwischen NutzerInnen. Zur Unterstreichung dieser Beobachtung wird auf die InteressenvertreterInnen verwiesen, die die Straße des 17. Juni quer durch den Tiergarten weiterhin als Eventmeile mit großem Stadtmarketingfaktor inszenieren und die die gartendenkmalpflegerischen Vorstellungen fast gänzlich dominieren würden. »Das sind natürlich die Bilder, die um die Welt gehen ... Vorne das Brandenburger Tor, hinten die Siegessäule« (C22). Mit den Events kann der Tiergarten bewirtschaftet werden, es fließen Gelder, die aber nicht im Gegenzug für die Pflege genutzt werden. »Da veranstaltet der Senat Marathonrennen, kassiert die Einnahmen irgendwo in ominöse Löcher und die Schäden im Park bleiben zurück«, in der blinden Annahme, »der Tiergarten ist ja völlig intakt, das macht ihm ja gar nichts« $(\mathrm{C} 21,22)$. Die NaturschützerInnen wiederum würden möglichst geringe Pflegeeingriffe fordern und damit eine Allianz mit dem Senat eingehen, der dieses Vorgehen mit einem wünschenswert geringen Budget verbinden kann. Die nicht zweckgebundenen Haushaltsmittel für die Grünpflege würden gerne genutzt, um andere Haushaltslöcher zu stopfen. Es gibt keine/n ParkdirektorIn (und keine klare Hierarchie), der/die ohne nennenswerte Interessenkonflikte für den Großen Tiergarten einsteht. »Das Einzige, was wirklich hilft, wäre entweder eine Stiftung für historische Parkanlagen in Berlin, ... mit richtigem Parkdirektor mit einem eigenen Budget und mit einer Zuständigkeit für alles, was mit dem Thema zu tun hat, auch das Veranstaltungsmanagement, die Parkpflege, die Kommunikation nach außen und und und « (C23, entsprechend J9). »Aber dass es den Politikern so egal ist, das ist halt dramatisch « (C18).

Für die Nutzerebene wird von den Gesprächspartnerinnen und -partnern ein ähnliches Machtungleichgewicht zwischen Interessen beschrieben. Das Machtvakuum ermögliche die Aneignung bestimmter Areale durch einzelne Nutzungsgruppen und gehe mit dem Exkludieren anderer einher. Beispielsweise entzögen sich bestimmte Areale mit Prostitution, mit Drogenhandel oder mit anderen Subkulturen der allgemeinen Nutzung als öffentlicher Raum (verbunden mit dem Verweis auf jahrhundertealte (kognitiv-kulturelle) Pfadabhängigkeiten zur liberalen Nutzung des Großen Tiergartens). GärtnerInnen würden sich zunehmend weigern, in bestimmten Bereichen des Gartens noch pflegerisch tätig zu werden, denn sie fühlten sich bedroht und als »Müllsammler« degradiert (I2, C29). Die fehlenden direkten AnwohnerInnen und ihr geringer Organisationsgrad im Sinne eines »Vereins der Freunde des Großen Tiergartens e.V.« würden ein Übriges zur Sache tun (I2). Auch starke Naturschutzinteressen würden den Erhalt des Gartendenkmals erschweren, etwa wenn sie sich durch den Schutz des Röhricht im Berliner Naturschutzgesetz eine rechtlich zugesicherte Dominanz über die Ideen einer dazu konträren Gartendenkmalpflege sichern (NatSchG Bln 2013, \$29ff ). Die Idee einer liberalen Nutzung steht im Gegensatz zu den Notwendigkeiten gartendenkmalpflegerischer Arbeiten für den Erhalt als historischer Garten. Die Interessen des Großen Tiergartens als Gartendenkmal werden demnach nicht adäquat auf politischer Ebene vertreten. 
Insgesamt zeichnen die InterviewpartnerInnen das Bild einer politischen Embeddedness, in der sich die Machtverteilung zuungunsten des Großen Tiergartens fast hoffnungslos verfestigt hat. Die Wahrnehmung als Stadtpark statt als Gartendenkmal dominiere (C24).

In diese Beschreibung wird auch die Bedeutung des Themas Klimawandel eingeordnet. Zwar werden etwa die längeren Trockenperioden klar ursächlich dem Klimawandel zugeschrieben. Er wird aber lediglich als ein Problem des Großen Tiergartens unter vielen anderen wahrgenommen. »Meine Theorie ist, dass man erstmal die Vegetation so pflegen muss, wie man sie auch ohne Klimawandel pflegen müsste« (C34). »Es wird viel darüber diskutiert, welche Baumarten dem Klimawandel trotzen können. Aber vorher sollte die Stadt erstmal Standortbedingungen schaffen, damit die Vegetation überhaupt überleben kann« (I3). Dies unterstützt unsere These, dass das Thema Klimawandel erst konstruktiv diskutiert werden kann, wenn im jeweiligen historischen Garten der grundlegende Bedarf für Pflege oder Finanzierung gedeckt ist. Gleichzeitig bietet der Klimawandel für die historischen Gärten auch eine Chance, durch seine Aktualität notwendige Ressourcen für den von ihm unabhängigen Pflege- und Finanzierungsbedarf zu mobilisieren.

\section{Fazit und Ausblick}

Die Resilienz historischer Gärten in Zeiten des Klimawandels hängt zweifelsohne von naturwissenschaftlichen Konzepten und einer angemessenen finanziellen und personellen Ausstattung ab. Im Sinne einer komplementären Betrachtung der Chancen auf Umsetzung angedachter Konzepte wollen wir auf die Potentiale einer soziologischen Perspektive aufmerksam machen. Die Analyse der Situation historischer Gärten im Hinblick auf ihre jeweilige kognitive, kulturelle, strukturelle und politische Embeddedness zielt auf eine Bestandsaufnahme ermöglichender oder begrenzender Bedingungen. Je stärker in der jeweiligen Embeddedness-Dimension die ermöglichenden die begrenzenden Einflüsse überwiegen, desto resilienter dürfte die jeweilige »Organisation historischer Gärten« auch auf Krisen und Herausforderungen, nicht nur des Klimawandels, reagieren können.

Eine unserer Beobachtungen ist, dass es eine Korrelation zu geben scheint zwischen einer bewussten Wahrnehmung und Pflege der organisationalen Umwelt und der Sensibilität für anstehende Herausforderungen wie den Klimawandel. Letztlich heißt dies, die Gartenverantwortlichen benötigen die Fähigkeit und die Ressourcen, sich auf ihre Umwelt, konkret die vier Embeddedness-Dimensionen, bewusst einzulassen. Ein aktives Stakeholder-Management ist dabei nur ein Aspekt. Die Führungskräfte brauchen auch hinreichend Freistellung vom operativen Geschäft, um dieser Aufgabe angemessen nachgehen zu können; und sie brauchen auch hinreichend Irritation, um die eigenen kognitiven Begrenzungen und kulturellen Limitation bewusst hinterfragen zu können, beispielsweise durch mehr Diversität in der eigenen Organisation.

Was wir zudem feststellen konnten, sind große Differenzen im Hinblick darauf, wie historische Gärten den Klimawandel wahrnehmen und angehen. Obwohl alle Gärten in Ost- 
deutschland zukünftig mit vergleichbaren klimatischen Herausforderungen zu rechnen haben, zeigt sich bei der Antwort der Gartenverantwortlichen auf diese anstehenden Aufgaben eine überraschend große Spannbreite. Während manche Gärten das Problem proaktiv angehen, den Klimawandel bewusst nach innen wie nach außen thematisieren oder die Wissenschaft gezielt mit einbinden, sind andere Gärten eher abwartend oder sogar regelrecht defensiv. Das ist ein erstaunlicher und auch von uns nicht erwarteter Befund mit weitreichenden Konsequenzen für die Entwicklung möglicher Resilienz-Strategien. Dort, wo der Klimawandel nur bedingt als Herausforderung oder Problem wahrgenommen wird, werden auch technische Lösungsansätze auf Skepsis stoßen oder ins Leere laufen. Wie im Beitrag gezeigt, verorten wir die Erklärung für diese signifikanten Unterschiede in der jeweils unterschiedlichen Embeddedness der historischen Gärten. Jeder Garten ist mit unveränderlichen Merkmalen auf den vier Ebenen der Einbettung konfrontiert, aber auch mit Gestaltungsspielräumen etwa in den Werten, Einstellungen oder Normen. Dieses Phänomen haben die historischen Gärten mit allen anderen Organisationen gemein und zeichnet sie nicht als Sonderfall aus. Vielleicht ist es aber der besondere Zauber der historischen Gärten, der das Bauen von Brücken zwischen gegensätzlichen Positionen einfacher macht als in anderen Feldern.

\section{Literaturverzeichnis}

Bandelj, Nina (2009): Emotions in Economic Action and Interaction. In: Theory and Society 38.4, 347-366.

Dacin, M. Tina; Ventresca, Marc J.; Beal, Brent D. (1999): The embeddedness of organizations: Dialogue \& Directions. In: Journal of Management 25.3, 317-356.

Deppisch, Sonja (2016): Urbane sozial-ökologische Resilienz. In: Multidisziplinäre Perspektiven der Resilienzforschung. Studien zur Resilienzforschung. Hg. von Rüdiger Wink. Wiesbaden: Springer Verlag, 199-213.

Granovetter, Mark (1985): Economic action and social structure: the problem of embeddedness. In: American Journal of Sociology 91.3, 481-510.

Iversen, Roberta R.; Armstrong, Annie Laurie (2008): Hurricane Katrina and New Orleans: What might a sociological embeddedness perspective offer disaster research and planning? In: Analyses of Social Issues and Public Policy 8.1, 183-209.

March, James G.; Simon, Herbert A. (1958): Organizations. Oxford: Wiley.

Mayring, Philipp (2015): Qualitative Inhaltsanalyse: Grundlagen und Techniken. 12. aktualisierte Auflage. Weinheim: Beltz Pädagogik.

Scott, W. Richard; Davis, Gerald F. (2007): Organizations and Organizing. Rational, Natural and Open Systems Perspectives. Upper Saddle River, NJ: Pierson Prentice Hall.

Thompson-Dyck, Kendra; Mayer, Brian; Anderson, Kathryn F.; Galaskiewicz, Joseph (2016): Bringing People Back In - Crisis Planning and Response Embedded in Social Contexts. In: Urban Resilience - A Transformative Approach. Hg. von Yoshiki Yamagata und Hiroshi Maryama. Basel: Springer International Publishing Switzerland, 279-293.

Uzzi, Brian (1996): The sources and consequences of embeddedness for the economic performance of organizations: the network effect. In: American Sociological Review 61.4, 674-698.

Zukin, Sharon; DiMaggio, Paul (1990): Introduction. In: Structures of Capital: The Social Organization of the Economy. Hg. von Sharon Zukin und Paul DiMaggio. Cambridge: Cambridge University Press, 1-36. 